\title{
次元の異るベクトル量の相関係数。
}

\author{
小河原 正已
}

\section{\$1. 緒言}

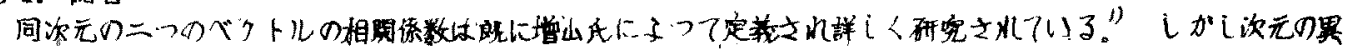

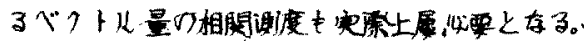

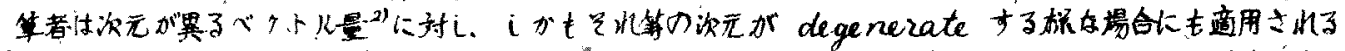

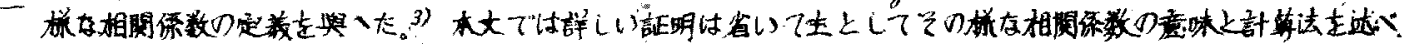

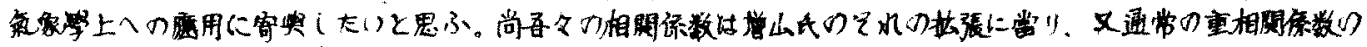

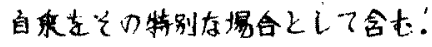

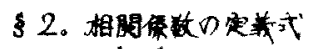

1)先づ蔽罩的点に

$$
x=\left(\begin{array}{c}
x_{1} \\
x_{2} \\
\vdots \\
x_{m}
\end{array}\right) \quad, \quad y=\left(\begin{array}{c}
y_{1} \\
y_{2} \\
\vdots \\
y_{n}
\end{array}\right)
$$

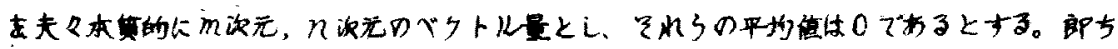

$$
\bar{x}_{i}=0,(i=1.2, \cdots, m) ; \quad \bar{y}_{i} \doteq 0,(i=1,2, \cdots, n)
$$

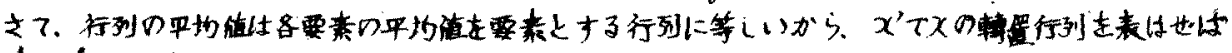

$$
\overline{x^{\prime}}=-\left(\begin{array}{ccc}
\overline{x_{1} x_{1}} & \cdots & \overline{x_{1} \bar{x}_{m}} \\
\cdots \cdots & \cdots & \ldots \\
\overline{x_{m} x_{1}} & \cdots & \overline{x_{m} x_{m}}
\end{array}\right) \text {. }
$$

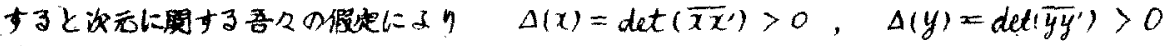

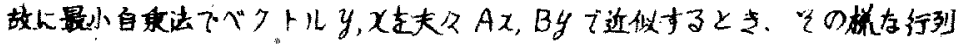

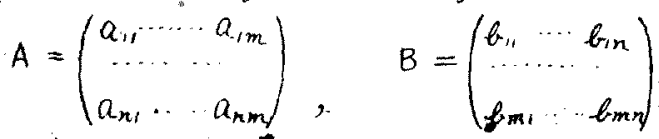

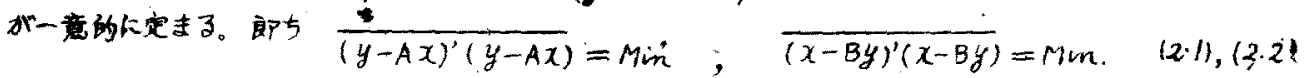

$$
\text { かj } \quad A=\left(\overline{y x^{\prime}}\right)\left(\overline{x x^{\prime}}\right)^{-1}, \quad B^{\prime}=\left(\overline{x y^{\prime}}\right)\left(\overline{y y^{\prime}}\right)^{-1} \quad \text { (2.3), (2.4) }
$$

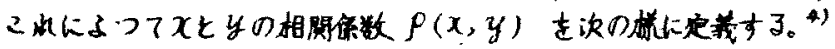

$$
\rho(x, y)=\frac{S_{k}(A B)}{\operatorname{Min} .(\min )}
$$

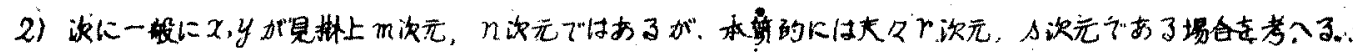

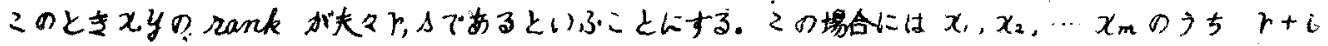

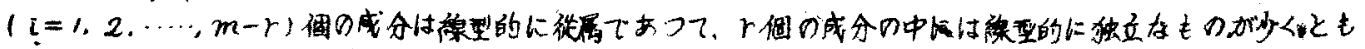

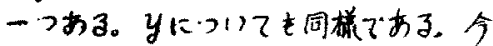

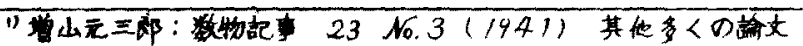

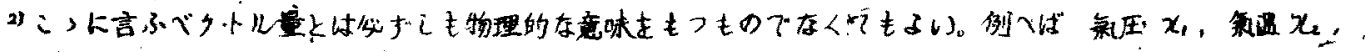

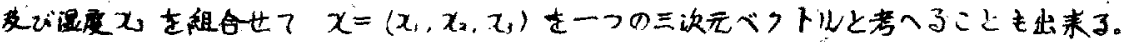

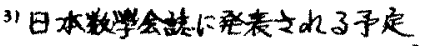

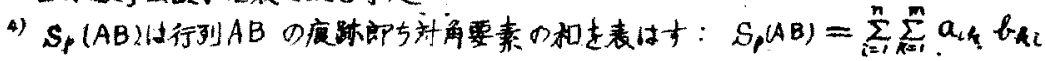




$$
x^{*}=\left(\begin{array}{c}
x_{1} \\
x_{2} \\
\vdots \\
x_{r}
\end{array}\right), \quad y^{*}=\left(\begin{array}{c}
y_{1} \\
y_{2} \\
\vdots \\
y_{s}
\end{array}\right)
$$

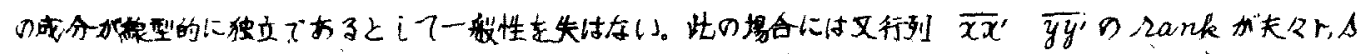

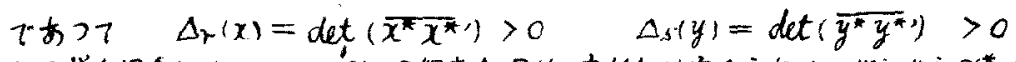

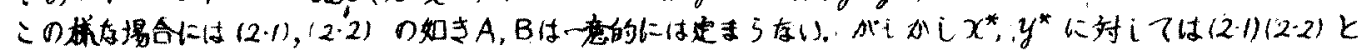

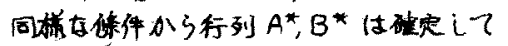

$$
S_{p}(A B)=S_{p}\left(A^{*} B^{*}\right)
$$

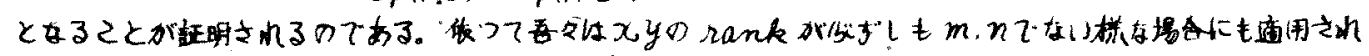
るものとし?

$$
\rho, x, y)=\frac{s_{p}(A B)}{\operatorname{Min}(\operatorname{rank}(x) \cdot \operatorname{rank}(y))} \quad(2, j)
$$

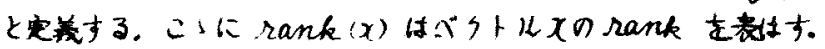

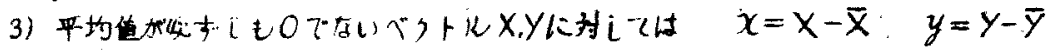

$$
\begin{aligned}
& f(x, y)=p(x, y) \text {. (2.8) }
\end{aligned}
$$

とするととは占ふまでをむい。

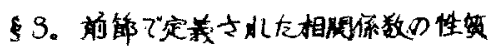

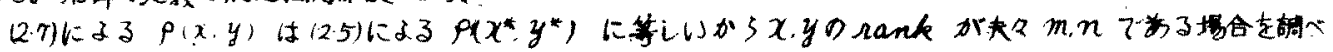

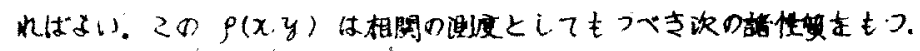

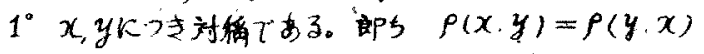

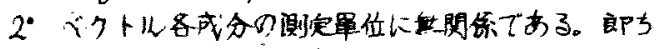

$$
x=\left(\begin{array}{cc}
\alpha_{1} & 0 \\
0 & \alpha_{m}
\end{array}\right) \quad \beta=\left(\begin{array}{cc}
\beta_{1} & 0 \\
0 & \beta_{n}
\end{array}\right)
$$

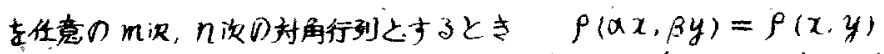

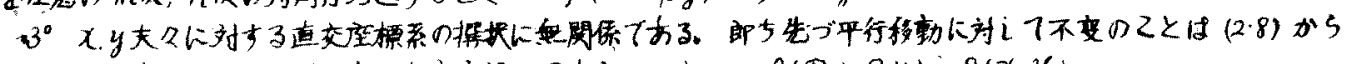

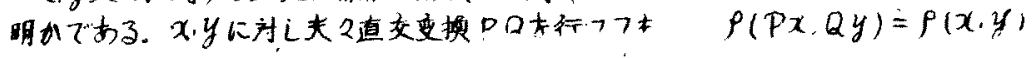

$4^{\circ} 0$ と，况用の实数でる。即士 $0 \leqq \rho(x, y) \leqq 1$

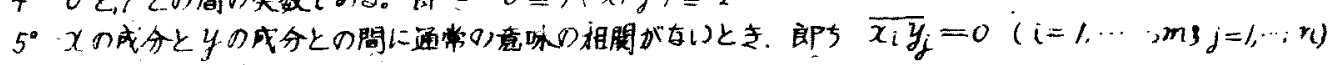
のと文，又的時に限つて $P(x, y)=0$

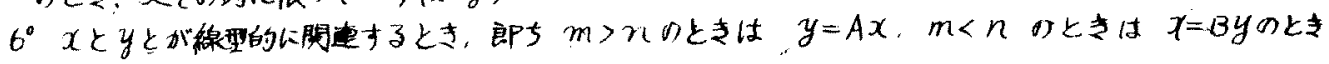
x资时に很つ? $P(x, y)=1$,

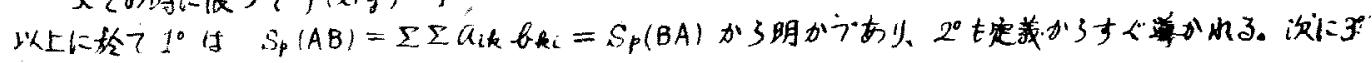

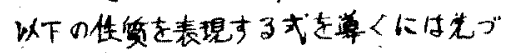

$$
A B=E_{n}-\frac{1}{\Delta(x)}\left(R^{i j}\right)\left(\overline{y y^{\prime}}\right)^{-1}, \quad \text { 或/d } \quad B A=E_{m}-\frac{1}{\Delta(y)}\left(S^{i j}\right)\left(\overline{x x^{\prime}}\right)^{-1} \quad(3 \cdot 1),(3 \cdot 2)
$$

と变形する。こいた

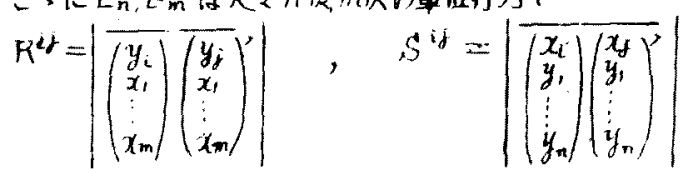

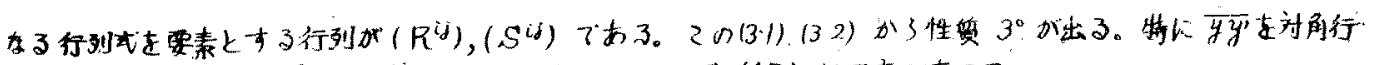

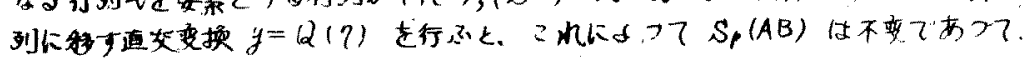




$$
S_{p}(A B)=n-\left\{\frac{\vec{R}^{\prime \prime}}{\eta_{1} \eta_{1} \Delta(x)}+\frac{\bar{R}^{2 p}}{\eta_{n, 2} \Delta(x)}+\cdots+\frac{\bar{R}^{n n}}{\eta_{n} \eta_{n} \Delta(x)}\right\}
$$

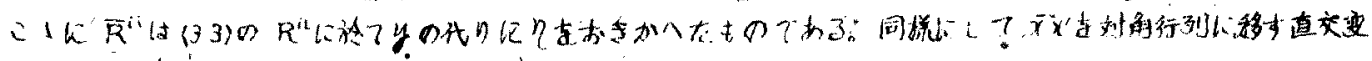
顺 $x=P(\xi)$ k小?

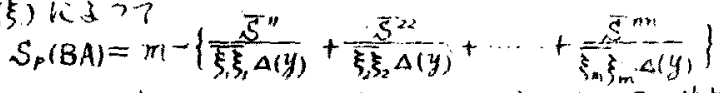

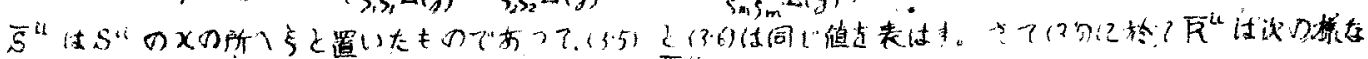

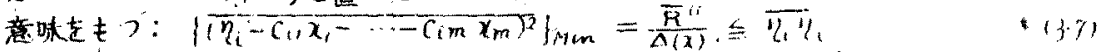

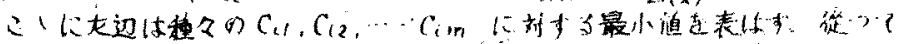

$$
0 \leq \frac{\bar{\pi}^{i 2}}{\eta_{i} \Delta(x)} \leqq 1
$$

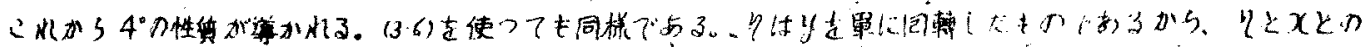

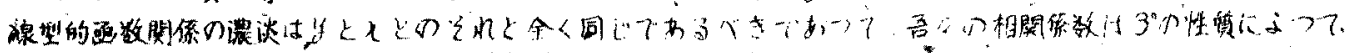

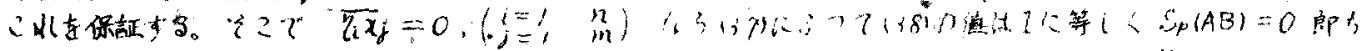

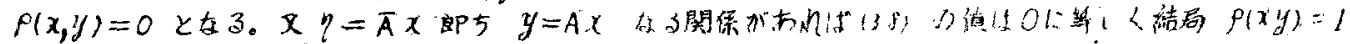

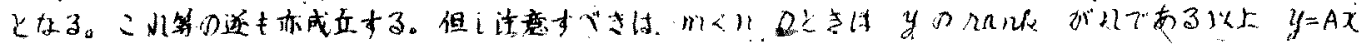

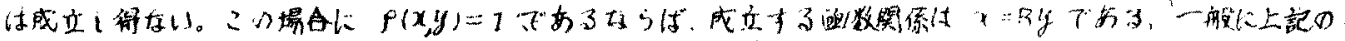

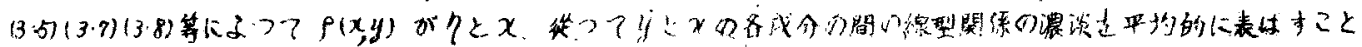
加分了。

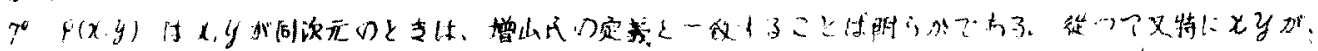

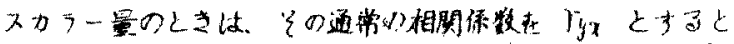

$$
f(x y)=\bar{x} \bar{y}^{2} / \bar{x} x y y=r_{y x}^{2} \quad 1.9 .9
$$

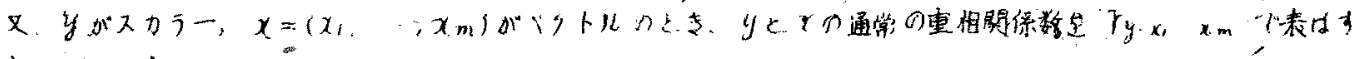
と(3-1)

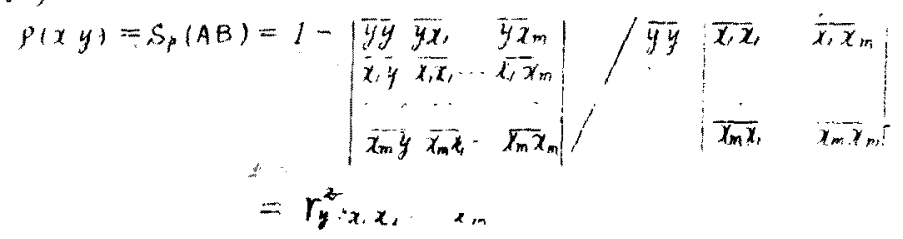

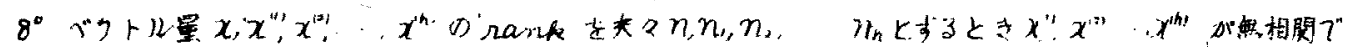

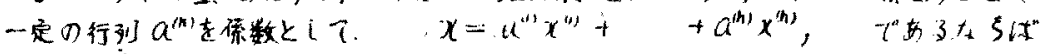

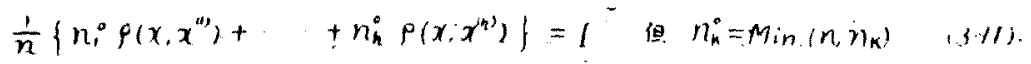

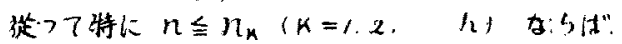

$$
f\left(x, x^{(1)}\right)+\cdots+f\left(x, x^{(n)}\right)=1 \quad \text { is }(2)
$$

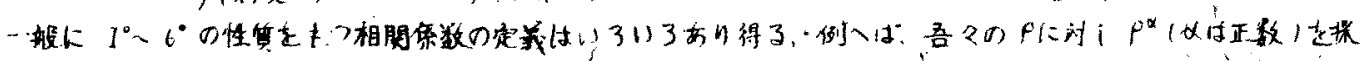

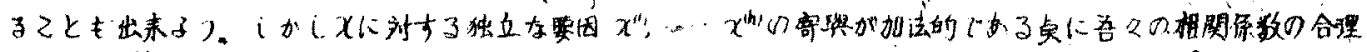
性がある。"

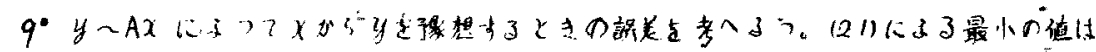

$$
D_{x}^{2}(y)=(y-A x)^{\prime}(y-A x)=\frac{R^{\prime \prime}}{\Delta(x)}+\frac{R^{\prime \prime}}{\Delta(x)+\cdots+\frac{R^{n n}}{A(x)} \quad(y / x)}
$$

となり, 一字 (3.5) か

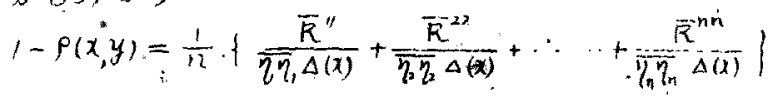

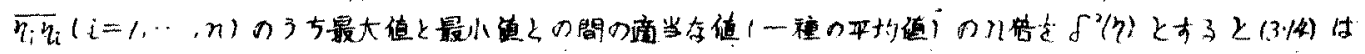

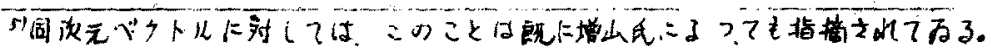




$$
1-\rho(x y)=\frac{1}{\delta^{2}(\eta)}\left\{\frac{\overline{R^{n}}}{\Delta(x)}+\cdots \cdot \cdots+\frac{\bar{R}^{n n}}{\Delta(x)}\right\}
$$

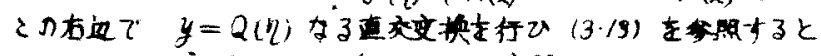

$$
D_{x}^{2}(y)=(1-\rho(x \cdot y)) \delta^{2}(y)
$$

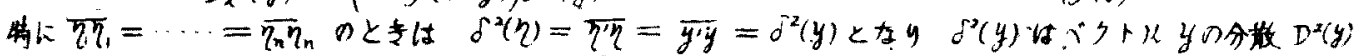

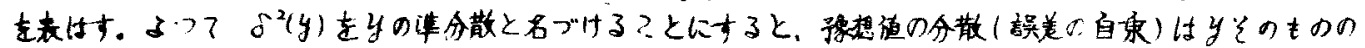

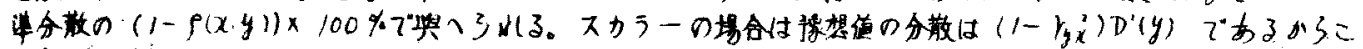

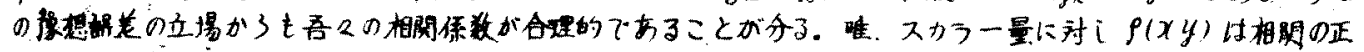

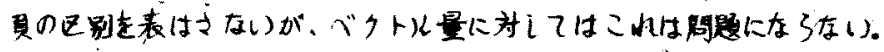

\section{4. 胡算法}

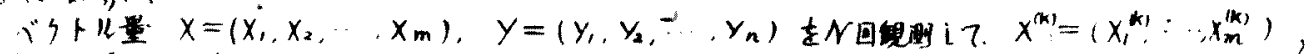

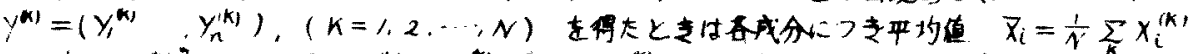

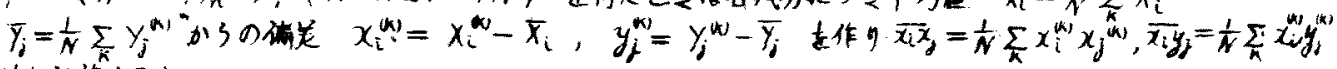
策を竍博なると

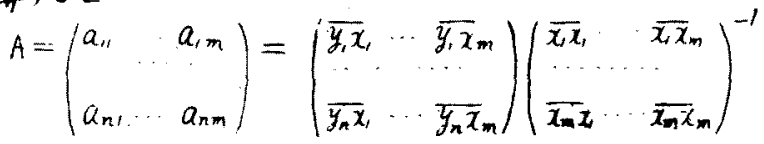

$$
\begin{aligned}
& B=\left(\begin{array}{ccc}
f_{11} & \ldots & b_{1 n} \\
\cdots & \ldots & \\
b_{m} & \ldots & b_{m n}
\end{array}\right)=\left(\begin{array}{lll}
\overline{x_{1} y_{1}} & \ldots & \overline{x_{1} y_{n}} \\
\overline{x_{m} y_{1}} & \cdots & \overline{x_{m} y_{n}}
\end{array}\right) \cdot\left(\begin{array}{ccc}
\overline{y_{1} y_{1}} & \ldots & \overline{y_{1} y_{n}} \\
\overline{y_{n} y_{1}} & \cdots & \overline{y_{n} y_{n}}
\end{array}\right)^{-1} \\
& S_{i}(A B)=\sum_{k=1}^{m} \sum_{i=1}^{n} a_{L x} \cdot G_{x_{i}} \quad \rho(x y)=\frac{S_{p}(A B)}{\operatorname{Min} \cdot(m, n)}
\end{aligned}
$$

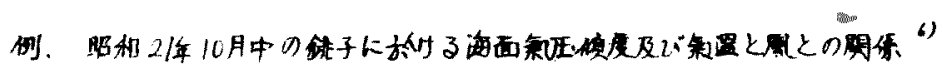

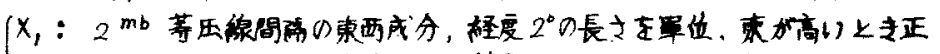

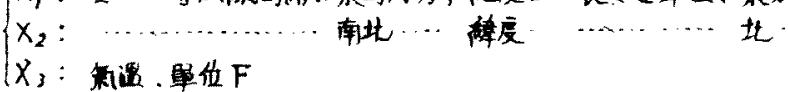

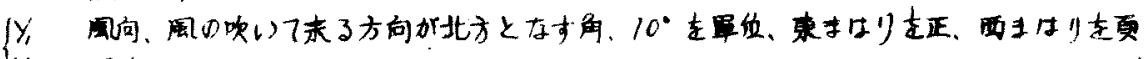
$y_{2}:$ Bufort

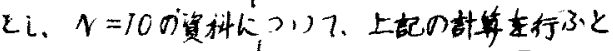

$$
\begin{aligned}
& \bar{X}=\left(\begin{array}{ccc}
0.35 & 0.46 & 67.9
\end{array}\right), \quad \bar{\gamma}=(3.9,4.0) \\
& A=\left(\begin{array}{ccc}
3.299 & -9.128 & 0.335 \\
-0.185 & -1.847 & -0.075
\end{array}\right), \quad B=\left(\begin{array}{ccc}
0.096 & -0.177 \\
-0.066 & -0.064 \\
0.647 & -1.314
\end{array}\right) . \\
& A B=\left(\begin{array}{cc}
1.013 & * \\
* & 0.232
\end{array}\right) \quad \text { 但 } i * 9 \text { 要来は不要 }
\end{aligned}
$$

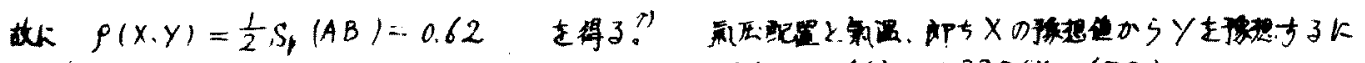
It At使つ7 $\left\{\begin{array}{l}y_{1}=3.9+3.299\left(x_{1}-0.55\right)-9.128\left(x_{2}-0.46\right)+0.335\left(x_{3}-67.9\right) \\ y_{2}=4-0.085\left(x_{1}-0.35\right)-7.847\left(x_{2}-0.46\right)-0.075\left(x_{3}-67.9\right)\end{array}\right.$

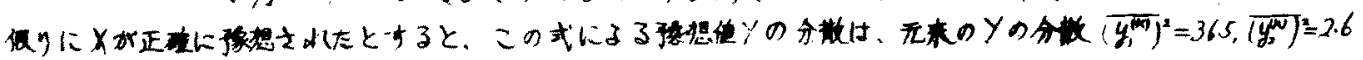

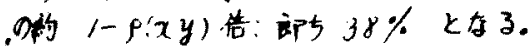

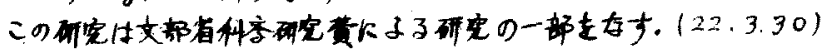

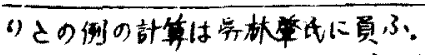

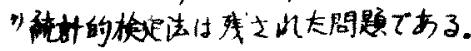

